PROCEEDINGS OF THE

AMERICAN MATHEMATICAL SOCIETY

Volume 127, Number 11, Pages 3229-3235

S 0002-9939(99)05086-8

Article electronically published on May 4, 1999

\title{
LUSIN'S THEOREM FOR DERIVATIVES WITH RESPECT TO A CONTINUOUS FUNCTION
}

\author{
V. AVERSA AND D. PREISS
}

(Communicated by Frederick W. Gehring)

Dedicated to Resia

\begin{abstract}
For a nowhere constant continuous function $g$ on a real interval $I$ and for a Borel measure $\mu$ on $I$, we give simple necessary and sufficient conditions guaranteeing, for any Borel function $f$ on $I$, the existence of a continuous function $F$ on $I$ such that the derivative of $F$ with respect to $g$ is, $\mu$ almost everywhere, equal to $f$.
\end{abstract}

Let $I$ be an interval of the real line $\mathbf{R}$ and let $g$ be a continuous function on $I$; we shall always assume that $g$ is non-constant on any subinterval of $I$. If $F$ is a continuous real-valued function on $I$, the natural notion of a derivative of $F$ with respect to $g$ at a point $x \in I$ is obtained by defining it as the number $F_{g}^{\prime}(x)$ having the property that for every $\varepsilon>0$ there is $\delta>0$ such that for every $|y-x|<\delta$,

$$
\left|F(y)-F(x)-F_{g}^{\prime}(x)(g(y)-g(x))\right| \leq \varepsilon|g(y)-g(x)| .
$$

The approach defining the derivative as a limit has also been used (see [1] and [3]); under the above continuity and non-constancy assumptions our definition is easily seen to be equivalent to

$$
F_{g}^{\prime}(x)=\lim _{y \rightarrow x} \frac{F(y)-F(x)}{g(y)-g(x)},
$$

where the limit is taken only over those $y$ for which $g(y) \neq g(x)$. It has also been suggested to define the ratio on the right side of (2) as zero whenever $y$ is such that $g(y)=g(x)$. This modification, however, leads to a different notion of derivative: If, for example, $g(y)=y \sin (1 / y), g(0)=0$, and $F=g$, then our definition gives $F_{g}^{\prime}(0)=1$, while the modified derivative does not exist. We intend to investigate to which extent can Lusin's theorem on existence of a.e. primitives to every Borel function be extended to derivatives with respect to $g$. The immediate obstacle is that an extension of this theorem in its original formulation, i.e., with Lebesgue measure, may be quite uninteresting because $g$ may well be related to a completely different measure. Since it may even happen that no measure is

Received by the editors January 22, 1998.

1991 Mathematics Subject Classification. Primary 26A36; Secondary 26A24, 28E99.

Key words and phrases. Relative derivatives.

The first author was supported by M.P.I fund.

The second author was a visiting Professor at Dipartimento di Matematica e Applicazioni dell'Università di Napoli. 
naturally associated with $g$, we decided to consider the (finite or, equivalently, $\sigma$ finite Borel) measure as being independent from the function, and seek conditions under which the analogy of Lusin's theorem holds. It turned out that a simple necessary and sufficient condition is that, after throwing away a set of $\mu$ measure zero, the inverse images of points under $g$ are composed of isolated points only. (See Theorem 3 for precise formulation.) It is not clear whether Lusin's theorem may hold with bigger exceptional $\sigma$-ideals of sets than those given by $\sigma$-finite Borel measures. In particular, this question may be of interest for the $\sigma$-ideal of sets of "peso nullo" (null weight), which was introduced by R. Caccioppoli [3] as a natural notion of exceptional sets in the theory of differentiation with respect to general continuous functions. With such possible future development in mind, we prove our main technical result, Proposition 1, in a more general formulation than needed for our application. We will need several preliminary results, some of them of independent interest. In their proofs we will sometimes use basic calculus of derivatives with respect to a function, like the rule for differentiating a finite sum or the product rule. These facts may be proved in the same way as their classical counterparts. Since the correct terminology, as "F is a continuous $\mu$-primitive to $f$ with respect to $g$ on an interval $I$," does not indicate the meaning of the notion well, we will adopt its slightly incorrect variants of the form " $\mathrm{F}$ is, $\mu$-a.e. in $I$, a continuous primitive to $f$ with respect to $g$." In any case, we mean that $F$ is a continuous function defined on $I$ such that $F_{g}^{\prime}(x)=f(x)$ for $\mu$ almost every $x \in I$. The assumptions of continuity and non-constancy of the function $g$ may be slightly relaxed. Such attempts have been made in certain special situations (see, e.g., [4] for a discussion of derivatives with respect to a possibly discontinuous monotonic function), but seem to be unnatural in the present development.

Theorem 1. Let $F$ and $g$ be two continuous functions on $I$. If $A=\{x \in I$ : $\left.F_{g}^{\prime}(x)=x\right\}$, then there exists a countable set $S$ such that for every $t \in \mathbf{R} \backslash S$ the set $g^{-1}(t) \cap A$ has only isolated points.

Proof. If $n \in \mathbf{N}$, we let $D_{n}$ denote the set of those $x \in I$ such that

$$
|F(y)-F(x)-x(g(y)-g(x))| \leq|g(y)-g(x)|
$$

whenever $|y-x| \leq \frac{1}{n}$. For every subset $D$ of $I$ we denote by $S_{D}$ the set of all $x \in D$ for which there is $\delta>0$ such that $g(y) \leq g(x)$ whenever $y \in D$ and $|y-x|<\delta$. Clearly, $g\left(S_{D}\right)$ is a countable set. Consequently,

$$
S=g\left(S_{I} \cup \bigcup_{n \in \mathbf{N}} S_{D_{n}}\right)
$$

is a countable set and thus, to prove the theorem, it suffices to show that $g^{-1}(t) \cap A$ has only isolated points for $t \notin S$. Let $x \in g^{-1}(t) \cap A$. Then there exists $n \in \mathbf{N}$ such that $x \in D_{n}$ and, since $x \notin S_{D_{n}}$, there is a sequence $x_{m}$ of points of $D_{n}$ tending to $x$ such that, for every $m=1,2, \ldots$,

$$
g\left(x_{m}\right)>g(x) .
$$

Suppose now that $y \in g^{-1}(t) \cap A, y \neq x$, and $|x-y|<\frac{1}{n}$. Let $y_{m} \in g^{-1}\left(g\left(x_{m}\right)\right)$ be the nearest point to $y$. Since $g(y)=t$ and $y \notin S_{I}$, we have that $y_{m} \rightarrow y$. For sufficiently large $m$ we infer from $x_{m} \in D_{n}$ and (3) that $F\left(y_{m}\right)=F\left(x_{m}\right)$; similarly from $x \in D_{m}$ we infer that $F(y)=F(x)$. Since also $g(y)=g(x)=t$ and 
$g\left(y_{m}\right)=g\left(x_{m}\right) \neq t$, we deduce that

$$
x=F_{g}^{\prime}(x)=\lim _{m \rightarrow \infty} \frac{F\left(x_{m}\right)-F(x)}{g\left(x_{m}\right)-g(x)}=\lim _{m \rightarrow \infty} \frac{F\left(y_{m}\right)-F(y)}{g\left(y_{m}\right)-g(y)}=F_{g}^{\prime}(y)=y,
$$

which is a contradiction.

The following lemmas are needed for our next theorem.

Lemma 1. Let $F$ and $g$ be continuous functions on $I$ and let $A$ be the subset of those points of $I$ at which $F$ has a derivative with respect to $g$. Then, for every $t \in \mathbf{R}$, the restriction of $F_{g}^{\prime}$ to $g^{-1}(t) \cap A$ is continuous.

Proof. Let $\varepsilon$ be a positive number and let $x$ be a point in $g^{-1}(t) \cap A$. Find $\delta>0$ such that, if $z \in I$ and $|z-x|<\delta$, then

$$
\left|F(z)-F(x)-F_{g}^{\prime}(x)(g(z)-g(x))\right| \leq \varepsilon|g(z)-g(x)| .
$$

If $y \in g^{-1}(t) \cap A$ and $|y-x|<\delta$, we find $z \in I$ such that $|z-y|<\delta-|y-x|$, $g(z) \neq g(y)$, and

$$
\left|F(z)-F(y)-F_{g}^{\prime}(y)(g(z)-g(y))\right| \leq \varepsilon|g(z)-g(y)|,
$$

and use that $g(y)=g(x)$ to infer that $F(y)=F(x)$ and

$$
\begin{aligned}
&\left|F_{g}^{\prime}(y)(g(z)-g(y))-F_{g}^{\prime}(x)(g(z)-g(y))\right| \\
&= \mid\left(F(z)-F(x)-F_{g}^{\prime}(x)(g(z)-g(y))\right) \\
&-\left(F(z)-F(y)-F_{g}^{\prime}(y)(g(z)-g(y))\right) \mid \\
& \leq \varepsilon|g(z)-g(x)|+\varepsilon|g(z)-g(y)| \\
&= 2 \varepsilon|g(z)-g(x)| .
\end{aligned}
$$

Dividing by $|g(z)-g(x)|$, we conclude that

$$
\left|F_{g}^{\prime}(y)-F_{g}^{\prime}(x)\right| \leq \varepsilon
$$

whenever $y \in g^{-1}(t) \cap A$ and $|y-x|<\delta$.

Lemma 2. Let $g$ be a continuous function on $I$ and let $\mu$ be a Borel measure on I. If every Borel function has, with respect to $g$, a continuous primitive, $\mu$-a.e. in $I$, then for every $t \in \mathbf{R}$ we have $\mu\left(g^{-1}(t)\right)=0$.

Proof. Suppose that there is $t \in \mathbf{R}$ with $\mu\left(g^{-1}(t)\right)>0$. Standard arguments show that there exist two disjoint Borel subsets $A$ and $B$ of $g^{-1}(t)$ having the same closure and such that for every open set $G$ meeting $\bar{A}$,

$$
\mu(G \cap A)>0 \quad \text { and } \quad \mu(G \cap B)>0 .
$$

Let $f$ be the characteristic function of the set $A$. By the assumption of the lemma, there exist a continuous function $F$ in $I$ and a $\mu$-null subset $X_{0}$ of $I$ such that $F_{g}^{\prime}(x)=f(x)$ whenever $x \in I-X_{0}$. According to Lemma 1 , the restriction of $f$ to $(A \cup B)-X_{0}$ is continuous, but this is impossible, since the sets $A-X_{0}$ and $B-X_{0}$ are both dense in $(A \cup B)-X_{0}$.

Theorem 2. Let $g$ be a continuous function on an interval $I$ and let $\mu$ be a Borel measure on I. If every Borel function has, with respect to $g$, a continuous primitive $\mu$-a.e. in $I$, then there exists a $\mu$-null subset $X_{0}$ of $I$ such that $g^{-1}(t) \cap\left(I-X_{0}\right)$ has only isolated points for every $t \in \mathbf{R}$. 
Proof. Let $C$ be a $\mu$-null subset of $I$ and let $F$ be a continuous function on $I$ such that $F_{g}^{\prime}(x)=x$ whenever $x \in I \backslash C$. Using Theorem 1, we find a countable set $S \subset \mathbf{R}$ such that $g^{-1}(t) \cap(I \backslash C)$ has only isolated points for $t \in \mathbf{R} \backslash S$. Since, according to Lemma $2, \mu\left(g^{-1}(t)\right)=0$ for every $t \in \mathbf{R}$, we infer that $\mu\left(g^{-1}(S)\right)=0$. Thus, the set

$$
X_{0}=C \cup g^{-1}(S)
$$

has $\mu$-measure zero and $g^{-1}(t) \cap\left(I \backslash X_{0}\right)$ has only isolated points for every $t \in \mathbf{R}$.

Lemma 3. Let $g$ be a continuous function on an interval $I, \mu$ a Borel measure on $I$, and $f$ a Borel function on $I$. Suppose that $X$ is a $\mu$-measurable subset of $I$ such that $g^{-1}(t) \cap X$ has only isolated points for every $t \in \mathbf{R}$. Then there exists a sequence $\left(P_{n}\right)$ of compact, nowhere dense, pairwise disjoint subsets of $X$ such that

(i) $\mu\left(X \backslash \bigcup_{n} P_{n}\right)=0$,

(ii) for every $n \in \mathbf{N}$, the restriction of $g$ to $P_{n}$ is one-to-one and the restriction of $f$ to $P_{n}$ is continuous, and

(iii) for every $n \in \mathbf{N}$ there is $\delta_{n}>0$ such that $g\left(P_{k}\right) \cap g\left(P_{n}\right)=\emptyset$ whenever $k \neq n$ and $\operatorname{dist}\left(P_{k}, P_{n}\right)<\delta_{n}$.

Proof. Let $r_{i}$ be a sequence dense in $I$ and let

$$
Q_{i, p}=\left\{x \in X \cap\left[r_{i}, r_{i}+1 / p\right]: g(y) \neq g(x) \text { whenever } y \in X,|y-x|<3 / p\right\} .
$$

Observing that $Q_{i, p}$ are $\mu$-measurable sets whose union is $X$, we use Lusin's Theorem to find nowhere dense disjoint compact sets $P_{i, p, k} \subset Q_{i, p}$ covering $\mu$-almost all of $X$ such that the restriction of $f$ to each $P_{i, p, k}$ is continuous. We observe that $\operatorname{dist}\left(P_{j, q, l}, P_{i, p, k}\right)<\max \{1 / p, 1 / q\}$ whenever $(j, q, l) \neq(i, p, k)$ and $g\left(P_{j, q, l}\right) \cap$ $g\left(P_{i, p, k}\right) \neq \emptyset$. Indeed, otherwise we assume $q \geq p$ and find $x \in P_{i, p, k}$ and $y \in P_{j, q, l}$ such that $g(x)=g(y)$. Since $y \neq x$ and $x \in Q_{i, p}$, we infer that $|y-x| \geq 3 / p$, which, together with the fact that the sets $P_{i, p, k}$ and $P_{j, q, l}$ have diameter not exceeding $1 / p$, gives $\operatorname{dist}\left(P_{j, q, l}, P_{i, p, k}\right) \geq|y-x|-2 / p>1 / p=\max \{1 / p, 1 / q\}$. Finally, we order the pairs $\left(P_{i, p, k}, 1 / p\right)$ into a sequence $\left(P_{n}, \delta_{n}\right)$, and note that all the required properties hold; the property (iii) follows from the above observation.

Lemma 4. Let $f$ be a continuous function on a compact nowhere dense subset $P$ of $\mathbf{R}$. Then for every $\varepsilon>0$ there is a continuously differentiable function $H$ on $\mathbf{R}$ such that $H^{\prime}(x)=f(x)$ for $x \in P$ and $|H(x)|<\varepsilon$ for every $x \in \mathbf{R}$.

Proof. We choose $K>0$ such that $P \subset]-K, K[$ and extend $f$ to a continuous function on $\mathbf{R}$ such that $f(x)=0$ for $|x| \geq K$. Let $F$ be the indefinite integral of this extended function $f$. Let $a_{1}<\cdots<a_{n}$ be such that $a_{1} \leq-K, a_{n} \geq K$, and the the oscillation of $F$ is less then $\varepsilon / 2$ on each of the intervals $\left[a_{i}, a_{i+1}\right]$. Let $\alpha$ be the indefinite integral of the function $x \mapsto \operatorname{dist}(x, P)$. Since $P$ is nowhere dense, $\alpha$ is strictly increasing, and we infer that the function

$$
G(x)= \begin{cases}F\left(a_{1}\right) & \text { if } x \leq a_{1}, \\ F\left(a_{i}\right)+\frac{F\left(a_{i+1}\right)-F\left(a_{i}\right)}{\alpha\left(a_{i+1}\right)-\alpha\left(a_{i}\right)}\left(\alpha(x)-\alpha\left(a_{i}\right)\right) & \text { if } x \in\left[a_{i}, a_{i+1}\right], \\ F\left(a_{n}\right) & \text { if } x \geq a_{n},\end{cases}
$$

is continuously differentiable and has derivative zero on $P$. We also note that $G\left(a_{i}\right)=F\left(a_{i}\right)$ and $G(x)$ lies between $F\left(a_{i}\right)$ and $F\left(a_{i+1}\right)$ whenever $x \in\left[a_{i}, a_{i+1}\right]$. Clearly, the function $H(x)=F(x)-G(x)$ is continuously differentiable and $H^{\prime}(x)=$ 
$f(x)$ for every $x \in P$. Moreover, $H(x)=0$ for $x=a_{i}$, from which, since the oscillation of $F$ as well as of $G$ on $\left[a_{i}, a_{i+1}\right]$ is less than $\varepsilon / 2$, we infer that $|H(x)|<\varepsilon$ for all $x \in\left[a_{1}, a_{n}\right]$. Since $H$ is equal to zero on each of the intervals $\left.]-\infty, a_{1}\right]$ and $\left[a_{n}, \infty[\right.$, we conclude that $|H(x)|<\varepsilon$ for all $x \in \mathbf{R}$.

Lemma 5. Let $P$ be a compact subset of $\mathbf{R}$ and let $G$ be an open set containing it. Then there is a continuous function $\varphi$, continuously differentiable with respect to $g$, such that $\varphi(x)=0$ for every $x \notin G$ and $\varphi(x)=1$ for $x \in P$.

Proof. We may assume that $G$ has only finitely many components, since we may delete from it all those components that do not meet $P$. Hence $G$ is the union of finitely many disjoint open intervals $] a_{j}, b_{j}\left[\right.$. Let $a_{j}<c_{j}<d_{j}<b_{j}$ be such that $g\left(c_{j}\right) \neq g\left(a_{j}\right)$ and $g\left(d_{j}\right) \neq g\left(b_{j}\right)$. Let $\xi$ and $\zeta$ be continuously differentiable functions such that $\xi_{j}\left(a_{j}\right)=\zeta_{j}\left(b_{j}\right)=0, \xi_{j}\left(c_{j}\right)=\zeta_{j}\left(d_{j}\right)=1$, and $\xi_{j}^{\prime}\left(a_{j}\right)=\xi_{j}^{\prime}\left(c_{j}\right)=$ $\zeta_{j}^{\prime}\left(b_{j}\right)=\zeta_{j}^{\prime}\left(d_{j}\right)=0$, The functions $\xi_{j} \circ g$ and $\zeta_{j} \circ g$ are continuously diffentiable with respect to $g$ with derivative $\xi_{j}^{\prime} \circ g$ and $\zeta_{j}^{\prime} \circ g$, respectively. It therefore suffices to define $\varphi(x)=0$ for $x \notin G, \varphi(x)=1$ for $x \in \bigcup_{j}\left[a_{j}, b_{j}\right], \varphi(x)=\xi_{j}(g(x))$ for $x \in\left[a_{j}, c_{j}\right]$, and $\varphi(x)=\zeta_{j}(g(x))$ for $x \in\left[d_{j}, b_{j}\right]$.

Lemma 6. Let $f$ be a continuous function on a compact nowhere dense subset $P$ of $\mathbf{R}$ and let $g$ be a continuous function on $\mathbf{R}$ such that $g(P)$ is nowhere dense. Then for every open set $G \supset P$ and every $\varepsilon>0$ there is a continuous function $F$, continuously differentiable with respect to $g$, such that

(i) $F_{g}^{\prime}(x)=f(x)$ for $x \in P$.

(ii) $|F(x)|<\varepsilon$ for all $x \in \mathbf{R}$, and

(iii) $F(x)=0$ whenever $x \notin G$.

Proof. We first find a bounded open set $G_{0} \supset P$ such that $\overline{G_{0}} \subset G$ and use Lemma 5 to find a continuous function $\varphi$, continuously differentiable with respect to $g$, such that $\varphi(x)=0$ for every $x \notin G$ and $\varphi(x)=1$ for $x \in G_{0}$. By Lemma 4 there is a continuously differentiable function $H$ such that $H^{\prime}(y)=f \circ g^{-1}(y)$ for $y \in g(P)$, $|H(y)|<\varepsilon$ for all $y \in \mathbf{R}$, and $H(y)=0$ if $y \notin G$. Since the derivative of $H \circ g$ with respect to $g$ is $H^{\prime} \circ g$ the function $F=\varphi H \circ g$ has the desired properties.

Proposition 1. Suppose that $g$ is a continuous function on an interval I which is not constant on any subinterval of $I$, that $f$ is a real-valued function on $I$, that $P_{1}, P_{2}, \ldots$ is a sequence of compact, nowhere dense subsets of $I$, and that $\delta_{n}$ is a decreasing sequence of positive numbers such that for every $n \in \mathbf{N}$,

(i) $g\left(P_{n}\right)$ is nowhere dense,

(ii) the restriction $f$ to $P_{n}$ is continuous, and

(iii) $g\left(P_{k}\right) \cap g\left(P_{n}\right)=\emptyset$ whenever $k>n$ and $\operatorname{dist}\left(P_{k}, P_{n}\right)<\delta_{n}$.

Then there is a continuous function $F$ on $I$ such that $F_{g}^{\prime}(x)=f(x)$ for every $x \in \bigcup_{n} P_{n}$.

Proof. Let $Q_{k}$ be the union of all those sets $P_{n}$ with $n<k$ for which $g\left(P_{n}\right) \cap g\left(P_{k}\right)=$ $\emptyset$. Since $g\left(P_{k}\right)$ and $g\left(Q_{k}\right)$ are disjoint compact sets and $g$ is continuous, there is an open set $G_{k}$ such that

$$
P_{k} \subset G_{k} \subset\left\{x: \operatorname{dist}\left(x, P_{k}\right)<\delta_{k} / 2\right\} \quad \text { and } \quad g\left(\overline{G_{k}}\right) \cap g\left(Q_{k}\right)=\emptyset .
$$


We put

$$
\lambda_{k}= \begin{cases}2^{-k} \min \left\{1, \operatorname{dist}^{2}\left(g\left(Q_{k}\right), g\left(G_{k}\right)\right\}\right. & \text { if } Q_{k} \neq \emptyset \\ 2^{-k} & \text { if } Q_{k}=\emptyset\end{cases}
$$

and infer that $\lambda_{k}>0$. We let $F_{0}=0$ and, using Lemma 6 , we recursively construct continuous functions $F_{k}$ having continuous derivative with respect to $g$ such that

(a) $\frac{d F_{k}}{d g}(x)=f(x)-\sum_{j=1}^{k-1} \frac{d F_{j}}{d g}(x)$ for every $x \in P_{k}$,

(b) $\left|F_{k}(x)\right|<\lambda_{k}$ for every $x \in \mathbf{R}$, and

(c) $F_{k}(x)=0$ whenever $x \notin G_{k}$.

Clearly, $F=\sum_{k=1}^{\infty} F_{k}$ is a continuous function, and we just need to show that $F_{g}^{\prime}\left(x_{0}\right)=f\left(x_{0}\right)$ whenever $x_{0} \in P_{n}$ for some $n \in \mathbf{N}$. Suppose, for the remaining part of the proof, that such $x_{0}$ and $n$ are fixed. We first note that, because of (a),

$$
\frac{d\left(\sum_{k=1}^{n} F_{k}\right)}{d g}\left(x_{0}\right)=\frac{d F_{n}}{d g}\left(x_{0}\right)+\sum_{k=1}^{n-1} \frac{d F_{k}}{d g}\left(x_{0}\right)=f\left(x_{0}\right) .
$$

Hence it remains to show that

$$
\frac{d\left(\sum_{k=n+1}^{\infty} F_{k}\right)}{d g}\left(x_{0}\right)=0
$$

To this end, let $\varepsilon>0$ be given and let $0<\delta<\delta_{n} / 2$ be such that $\left|g(x)-g\left(x_{0}\right)\right|<2^{n} \varepsilon$ whenever $\left|x-x_{0}\right|<\delta$. Suppose that $\left|x-x_{0}\right|<\delta$. If $k>n$ and $F_{k}(x) \neq 0$, then $x \in G_{k}$, which implies that $\operatorname{dist}\left(x, P_{k}\right)<\delta_{k} / 2 \leq \delta_{n} / 2$, so $\operatorname{dist}\left(P_{k}, P_{n}\right)<\delta_{n}$. Thus (iii) implies that $g\left(P_{k}\right) \cap g\left(P_{n}\right)=\emptyset$, which shows that $P_{n} \subset Q_{k}$. Consequently, $g\left(x_{0}\right) \in g\left(Q_{k}\right), g(x) \in g\left(G_{k}\right)$, and we infer from (b) that

$$
\left|F_{k}(x)\right| \leq 2^{-k} \operatorname{dist}^{2}\left(g\left(Q_{k}\right), g\left(G_{k}\right)\right) \leq 2^{-k}\left|g(x)-g\left(x_{0}\right)\right|^{2} .
$$

Noting that this inequality holds also if $k>n$ and $F_{k}(x)=0$, we infer that

$$
\left|\sum_{k=n+1}^{\infty} F_{k}(x)\right| \leq 2^{-n}\left|g(x)-g\left(x_{0}\right)\right|^{2} \leq \varepsilon\left|g(x)-g\left(x_{0}\right)\right|
$$

whenever $\left|x-x_{0}\right|<\delta$. We use this inequality first with $x=x_{0}$ to infer that $\sum_{k=n+1}^{\infty} F_{k}\left(x_{0}\right)=0$, and then with an arbitrary $\left|x-x_{0}\right|<\delta$ to conclude that

$$
\left|\sum_{k=n+1}^{\infty} F_{k}(x)-\sum_{k=n+1}^{\infty} F_{k}\left(x_{0}\right)\right| \leq \varepsilon\left|g(x)-g\left(x_{0}\right)\right|
$$

whenever $\left|x-x_{0}\right|<\delta$. By the definition of the derivative with respect to $g$, this shows (5).

Theorem 3. Let $g$ be a continuous function on an interval I which is not constant on any subinterval of $I$, and let $\mu$ be a Borel measure on I. Then every Borel function has, $\mu$-a.e. on $I$, a continuous primitive with respect to $g$ if and only if there exists a subset $X_{0}$ of $I$ such that $\mu\left(X_{0}\right)=0$ and $g^{-1}(t) \cap\left(I \backslash X_{0}\right)$ has only isolated points for every $t \in \mathbf{R}$.

Proof. Having proved the "only if" part in Theorem 2, we are left with the "if" part only. Let $f$ and $\mu$ satisfy the assumptions of the theorem. Using Lemma 3, we find a sequence $P_{1}, P_{2}, \ldots$ of compact, nowhere dense, pairwise disjoint subsets of $I \backslash X_{0}$ such that

(a) $\mu\left(I \backslash \bigcup_{n} P_{n}\right)=0$, 
(b) for every $n \in \mathbf{N}$, the restriction of $g$ to $P_{n}$ is one-to-one and the restriction of $f$ to $P_{n}$ is continuous, and

(c) for every $n \in \mathbf{N}$ there is $\delta_{n}>0$ such that $g\left(P_{k}\right) \cap g\left(P_{n}\right)=\emptyset$ whenever $k \neq n$ and $\operatorname{dist}\left(P_{k}, P_{n}\right)<\delta_{n}$.

We note that, since $P_{n}$ is a compact nowhere dense set on which $g$ is continuous and one-to-one, $g\left(P_{n}\right)$ is nowhere dense. Since we may clearly assume that the sequence $\delta_{n}$ is decreasing, we infer that all assumptions of Proposition 1 hold, and thus that the "if" part of the Theorem follows directly from Proposition 1.

\section{REFERENCES}

[1] V. Aversa, Sulla derivabilità delle funzioni di una variabile rispetto ad una funzione continua. Ric. Mat. 24, 182-198, 1975. MR 53:5815

[2] V. Aversa, Sugli insiemi di peso nullo rispetto ad una funzione continua Ric. Mat. 26, 79-84, 1977. MR 58:1070

[3] R. Caccioppoli, L'integrazione e la ricerca delle primitive rispetto ad una funzione continua qualunque. Ann. Mat. Pura e Appl. 40, 15-34, 1955. MR 17:954a

[4] M. Gradinaru, On the derivative with respect to a function with applications to RiemannStieltjes integral. Collection: Seminar on Mathematical Analysis (Cluj-Napoca, 1989-1990), 21-28, "Babes-Bolyai" Univ., Cluj-Napoca, 1990. MR 93g:26008

[5] J. Liberman, Théoréme de Denjoy sur la dérivée d'une fonction arbitraire par rapport á une fonction continue, Rec. Mathématique, 221-236, 9(51) 1941. MR 3:74d

Dipartimento di Matematica e Statistica, Via Cinzia, 80126 Napoli Italy

E-mail address: aversa@dmsna.dms.unina.it

Department of Mathematics, University College London, Gower Street, London WCIE-6BT, ENGLAND

E-mail address: dp@math.ucl.ac.uk 